\title{
Lignocellulose aerogel and amorphous silica nanoparticles from rice husks
}

\author{
Zichao Wei ${ }^{1,2}$, Andrew T. Smith ${ }^{1,2}$, William R. T. Tait ${ }^{2}$, Jingjing Liu ${ }^{1,2}$, Hao Ding ${ }^{1,2}$, Hui Wang ${ }^{1,3 *}$, Weixing Wang ${ }^{4}$ and \\ Luyi $\operatorname{Sun}^{1,2,5^{*}}$ (D)
}

\begin{abstract}
Rice Husks (RHs) are one of the most abundant sources of biomass in the world due to rice consumption. Lignocellulose and silica are two of the main components of $\mathrm{RHs}$, which allow RHs to be applied in different areas. Lignocellulose can be partially dissolved in 1-butyl-3-methylimidazolium chloride (BMIMCI), which is a simple way of competing with the traditional extraction methods that suffer from high chemical consumption. A lignocellulose freeze gel is obtained via a cyclic liquid nitrogen freeze-thaw (NFT) process. Multi-functional self-assembled lignocellulose aerogel is obtained after $\mathrm{CO}_{2}$ supercritical drying. Based on the aerogel's special properties, two routes are developed for practical applications. On one hand, the aerogel is coated to exhibit a superhydrophobic property that can be applied as an absorbent for oil spills. On the other hand, a carbon aerogel is synthesized via a pyrolysis process, resulting in a porous amorphous carbon. The residue after partially dissolving lignocellulose in $\mathrm{BMIMCl}$ is further calcined to obtain amorphous silica nanoparticles, achieving a comprehensive application of RHs.
\end{abstract}

Keywords: Rice husks, Lignocellulose, Carbon aerogel, Silica

\section{Introduction}

Rice husks (RHs) are one of the most abundant sources of biomass in the world because of the massive amount of rice consumed by the human population. There are two main components in RHs: silica (ca. 15-28 wt.\%) and lignocellulose (LC) (ca. 72-85 wt.\%) [1-3]. If isolated, the silica from RHs $[4,5]$ can be used to prepare various silicon-based materials, including silicon carbide [6], silicon nitride [7], silicon tetrachloride [8], silicon [9], and zeolite [10]. These materials have widespread applications in semiconductors [10], abrasive materials [10], pigments [11], catalyst supports [12], biomedicine [13], luminescence [14-17], etc. LC is a natural polymer, containing lignin, cellulose, and hemicellulose [18]. LC can be used to prepare biochemicals, such as bioethanol and xylitol [19], as well as quantum dots [20]. Of the three major contents of LC, cellulose is abundantly

\footnotetext{
* Correspondence: wanghui@scut.edu.cn; luyi.sun@uconn.edu

${ }^{1}$ Polymer Program, Institute of Materials Science, University of Connecticut, Storrs, CT 06269, USA

Full list of author information is available at the end of the article
}

available in nature, and has been used by humans for a wide range of applications for thousands of years [21]. Recently, cellulose has become an attractive sustainable resource and been applied across several areas, including cellulose gas barrier films [22], sensors [23], cellulose nanocrystals [23], and aerogels [24]. However, the extraction process of $\mathrm{RH}$ cellulose is very complex and time consuming [25]. Ionic liquids (ILs) were reported to be able to effectively dissolve cellulose and lignin [26, 27] because the ions in ILs can serve as the acceptor of hydrogen bonds, which helps to isolate the lignocellulose from RHs $[28,29]$. As such, ILs are a good working medium to directly extract LC from RH biomass, and dissolve those organic components to form a homogeneous system.

Aerogels are a group of materials with a 3-D porous network structure. Their ultra-low density, high surface area, and large porosity make them attractive for many applications, including catalyst supports [30], artificial muscles [31], supercapacitors [32], and absorption materials [33-37]. Carbon aerogels are particularly promising 
due to their high conductivity [38]. Graphene and carbon nanotubes from chemical vapor deposition (CVD), and resorcinol-formaldehyde aerogels derived from pyrolysis are the two main routes to prepare carbon aerogels $[39,40]$. However, the precursors are very expensive, and some of the chemicals used are also not friendly to the environment.

Since ILs are able to effectively dissolve LC from RHs [18], under a subsequent series of treatments of a liquid nitrogen freeze-thaw (NFT) process [41, 42], water regeneration, and $\mathrm{CO}_{2}$ supercritical drying, self-assembled $\mathrm{LC}$ aerogels can potentially be prepared [41]. In addition to their practical applications, such as absorption materials for various chemicals (e.g., oil spill cleanup) thanks to their high porosity and surface area, such self-assembled LC aerogels may also help researchers better understand the mechanism of the self-assembly of LC and collagen fibrils, two of the most abundant fibers in nature. How LC (predominately in plants) and collagen (predominately in animal bodies) fibrils self-assemble into larger scale structures in living body still remains a mystery [43].

Carbon aerogels can be further obtained via high temperature pyrolysis of LC aerogels under an inert atmosphere. The porous structure can be maintained and the entire organic components can be converted into carbon. Their remarkable chemical and physical properties make carbon aerogels promising for various applications, such as supercapacitors [44-46], catalyst supports $[47,48]$, and gas storage [48].

Biomass has recently become increasingly attractive because they are renewable. Both the inorganic and organic components in biomass can be potentially applied to different areas. But the major problem facing biomass utilization is the trade-off between efficiency and waste. For example, to extract high purity silica from RHs, traditionally the organic components are wasted and vice versa. Therefore, a route to derive both organic and inorganic components from RHs is highly describable and should be more sustainable.

Herein, we report a comprehensive strategy to prepare both LC aerogel and amorphous silica nanoparticles from RHs. The LC aerogel has a high surface area with a mesoporous structure. By surface modification with a hydrophobic agent, the aerogel with a hydrophobic surface was prepared. The carbon aerogel was further prepared by pyrolyzing the LC aerogel under an inert atmosphere. The residue from IL treated RHs was further calcined to prepare amorphous silica nanoparticles. These three processes demonstrate a good example to convert biomass into value added products, as well as the promising future of green chemistry.

\section{Results \& discussion}

The entire process for preparing self-assembled LC aerogel, carbon aerogel, and silica nanoparticles is shown in
Fig. 1. RHs, which contain a high content of LC, were partially dissolved in BMIMCl after $4 \mathrm{~h}$ of treatment at $85^{\circ} \mathrm{C}[18,28]$. The LC IL solution was obtained after centrifugation, and subsequently treated by the NFT process to induce gelation [22, 42]. First, the IL solution containing LC was frozen by liquid nitrogen, during which the IL solvent began to freeze, so that it contained a frozen and unfrozen liquid micro-phase [42]. After freezing for $6 \mathrm{~h}$, cellulose, hemicellulose, and lignin were extruded by the completely frozen IL crystalline. In this process, the gaps between cellulose macromolecules shrunk. The molecules were then crosslinked by physically twisted crosslinking points [42]. Finally, a gel formed during a slow thawing process due to an increasing strength of the physical crosslinking with an increasing temperature. By utilizing $\mathrm{CO}_{2}$ supercritical drying, a low-density LC aerogel was obtained. As shown in Fig. 1 , the self-assembled LC aerogel was light enough to stand on a dandelion. The density of the LC aerogel was measured to be $0.129 \mathrm{~g} / \mathrm{cm}^{3}$. This approach to prepare light aerogel is advantageous because of three reasons. First, the formation process does not use any crosslinking agent, which may cause environmental and health issues. Second, the process is very facile. The selfassembly thermodynamic process minimizes the utilization of chemicals. Third, RH biomass, sometime treated as a biowaste, was used as the staring material.

The cross section of the LC aerogel derived from RHs was characterized by SEM and the images are presented in Fig. 2a and b, which show that the LC IL solution formed a steady network structure with a dense porosity. The higher resolution SEM image indicates that the LC fibers interconnected at physical crosslinking points [22, 42]. The result suggests that the cyclic water regeneration could effectively remove IL, eliminating the barrier of the following gelation process. Overall, the NFT process successfully led to the formation of a hydrogel from the IL LC solution. With the assistance of $\mathrm{CO}_{2}$ supercritical drying, the hydrogel could maintain its porous network structure to form an aerogel, promising for a wide variety of applications depending on the porous structure [42].

Self-assembled LC aerogel is a porous material that contains an abundance of carbon. Thus, carbon aerogel could be generated from LC aerogel in an inert atmosphere under elevated temperatures. As shown in Fig. 2c and $d$, the porous network structure was well maintained throughout the high temperature pyrolysis, which indicates that the LC aerogel could maintain its structure at elevated temperatures. The inert atmosphere also contributed to minimizing the damage to the porous structure. The lignin and hemicellulose wrapped around cellulose were changed to spheres after pyrolysis and attached to the carbonized cellulose structure [49]. 

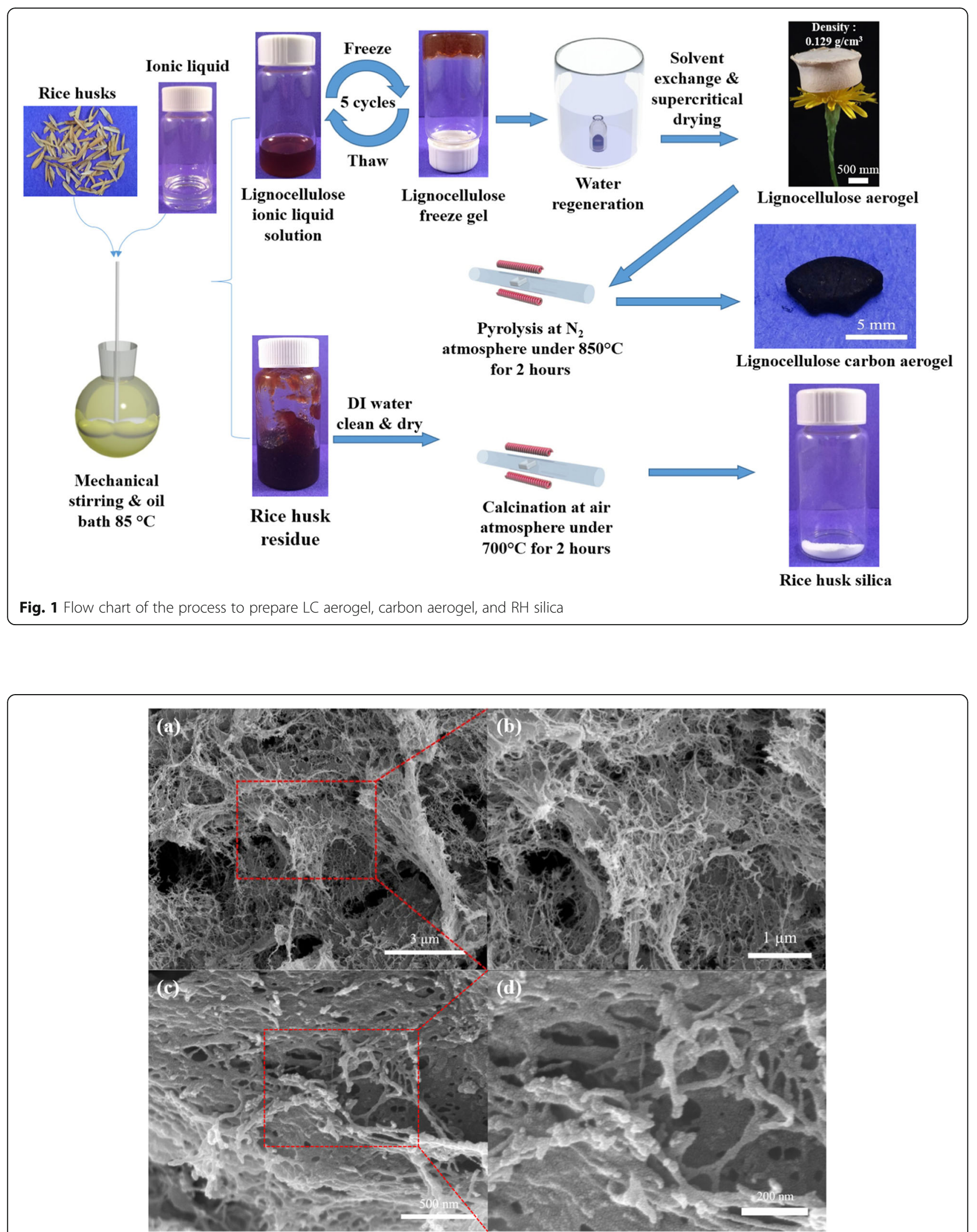

Fig. 2 a SEM image of the LC aerogel synthesized after 5 cycles of NFT; $\mathbf{b}$ enlarged area of Figure $\mathbf{b}$; c SEM images of the carbon aerogel prepared via the pyrolysis of LC aerogel under $1000^{\circ} \mathrm{C}$ for $2 \mathrm{~h}$; $\mathbf{d}$ enlarged area of Figure (c) 

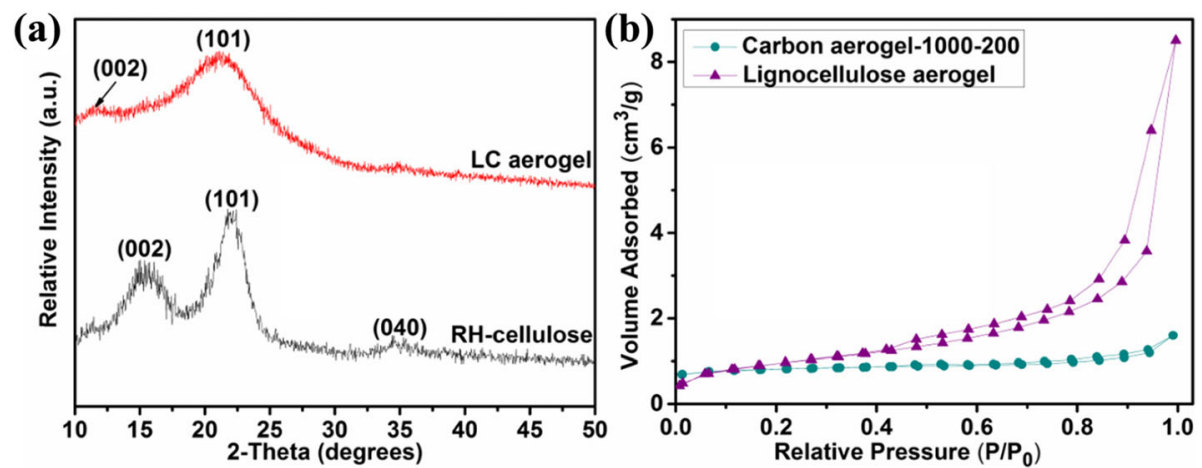

Fig. 3 a XRD patterns of the LC aerogel and the cellulose derived from $R H s ; \mathbf{b} \mathrm{N}_{2}$ adsorption-desorption isotherms of the LC aerogel and carbon aerogel

Figure 3a shows the XRD patterns of the LC aerogel and $\mathrm{RH}$ cellulose. The results show that $\mathrm{RH}$ cellulose bears the typical cellulose I structure according to the characteristic diffraction peaks (002), (101), and (040), at $15.5^{\circ}, 22.1^{\circ}$, and $34.7^{\circ}$, respectively [50]. The LC aerogel was obtained after water regeneration and $\mathrm{CO}_{2}$ supercritical drying. It mainly showed one broad hump at ca. $21.1^{\circ}$, indicating that the regenerated LC has a lower degree of crystallinity than the raw cellulose, which is expected [51]. According to the previous reports [52, 53], the regenerated cellulose or lignocellulose possess a cellulose II structure with a characteristic peak (002) at $12.1^{\circ}$, which is also shown in the XRD pattern of the LC aerogel. The lower crystallinity of the regenerated $\mathrm{LC}$ is due to some of the intrinsic inter- and intra-molecular hydrogen bonds and destruction of crystallized structures of cellulose in the dissolving process [51]. However, the intensity of the (002) peak of the LC aerogel is relatively low as shown in Fig. 3a, due to the fact that lignin restrained the swelling of cellulose molecules in IL and limited the solubility of cellulose molecules at relatively low temperatures $\left(85^{\circ} \mathrm{C}\right)[42,53,54]$.

As shown in Fig. 3b, the $\mathrm{N}_{2}$ adsorption-desorption curves of the LC aerogel and carbon aerogel prepared at $1000{ }^{\circ} \mathrm{C}$ are of type IV, suggesting that the two types of aerogels are mesoporous. The curves also indicate that the LC aerogel formed a mesoporous network structure after cyclic NFT thermodynamic crosslinking, and the carbon aerogel maintained a porous structure after pyrolysis. Based on the isotherms, the specific surface area (SSA) and pore property data were calculated and are summarized in Table 1 . The LC aerogel derived from
RHs has a relatively higher total specific surface area (SSA (total) of $106.5 \mathrm{~m}^{2} / \mathrm{g}$, compared to the literature data of $80.7 \mathrm{~m}^{2} / \mathrm{g}$ [22]. The main reason is most likely because RHs contain a high cellulose content [55], which could minimize the effect of lignin in the solution, and assist the formation of a porous network structure. The LC aerogel also has a high SSA (meso)/SSA (total) ratio, which is probably due to the low surface tension at the supercritical $\mathrm{CO}_{2}$ /hydrogel interface during $\mathrm{CO}_{2}$ supercritical drying. After the conversion of $\mathrm{LC}$ aerogel to carbon aerogel, the SSA (total) of the carbon aerogel sample reached $217.3 \mathrm{~m}^{2} / \mathrm{g}$.

LC is hydrophilic due to the hydroxyl groups present on the structures of lignin, cellulose, and hemicellulose. It was reported that a hydrophobic coating could be applied on a recycled cellulose aerogel to make it oleophilic [33]. One of the main incentives to convert cellulose aerogels to be hydrophobic is because hydrophobic aerogels can repel water and attract oil, making them promising for applications such as oil spill cleaning [56]. Figure $4 \mathrm{a}$ and $\mathrm{b}$ shows the water contact angles (WCAs) of $132.5^{\circ}$ and $130.5^{\circ}$ on an external and a freshly fractured surface of the coated LC aerogel, respectively. This result suggests that the hydrophobic modification by MTMS vapor can reach deep into the LC aerogel, and the entire 3-D porous structure was converted to be hydrophobic.

In order to investigate the potential oil absorption performance of the surface-modified LC aerogel, pump oil stained by Sudan III dye (to show a high visibility) was adopted for the demonstration, and the result is presented in Fig. 4c. The surface modified

Table 1 Surface area of the LC aerogel and carbon aerogel

\begin{tabular}{llllll}
\hline Sample & $\begin{array}{l}\mathbf{S S A}_{\text {(total) }} \\
{\left[\mathbf{m}^{2} / \mathbf{g}\right]}\end{array}$ & $\begin{array}{l}\mathbf{S S A}_{\text {(meso) }} \\
{\left[\mathbf{m}^{2} / \mathbf{g}\right]}\end{array}$ & $\begin{array}{l}\mathbf{S S A}_{\text {(micro) }} \\
{\left[\mathbf{m}^{2} / \mathbf{g}\right]}\end{array}$ & $\begin{array}{l}\mathbf{S S A}_{\text {(meso) }} / \mathbf{S S A}_{\text {(total) }} \\
{[\%]}\end{array}$ & $\begin{array}{l}\mathbf{S S A}_{\text {(micro) }} / \mathbf{S S A}_{\text {(total) }} \\
{[\%]}\end{array}$ \\
\hline LC aerogel & 106.5 & 100.2 & 6.2 & 94.1 & 5.8 \\
Carbon aerogel & 217.3 & 55.5 & 143.6 & 25.5 & 66.1 \\
\hline
\end{tabular}




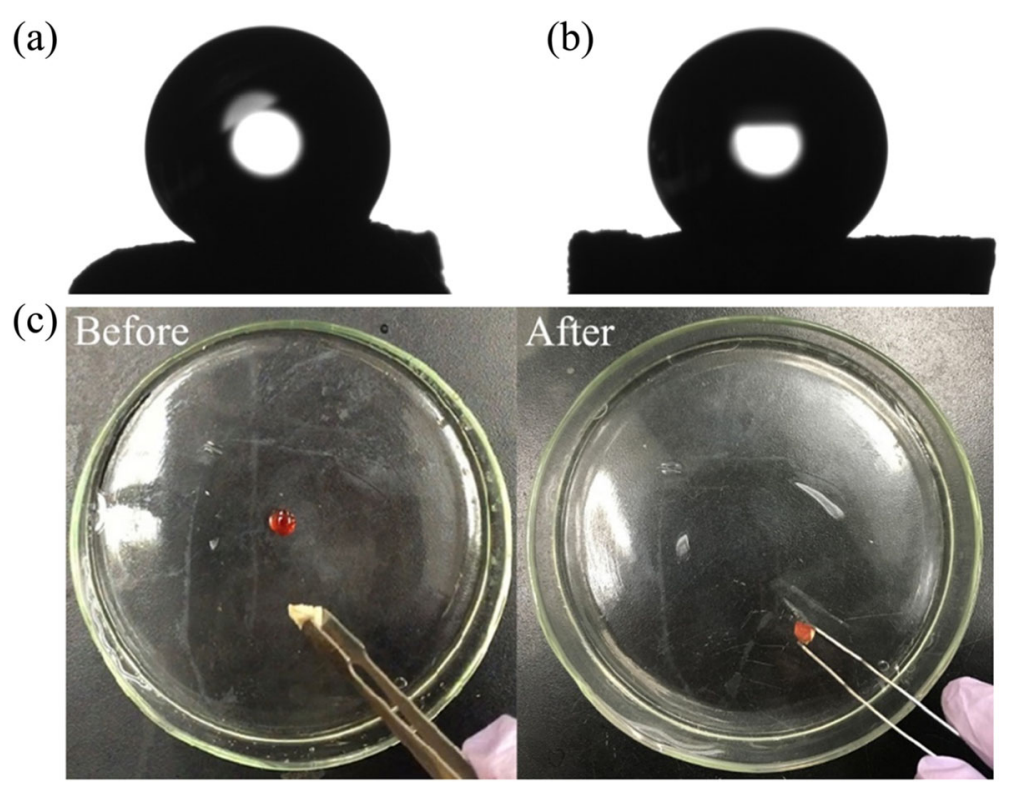

Fig. 4 Water contact angles of the MTMS vapor treated LC aerogel: a on an external surface, and $\mathbf{b}$ on a freshly fractured surface. $\mathbf{c}$ Pump oil absorption process by the MTMS vapor treated LC aerogel

LC aerogel can easily and quickly absorb the pump oil. At the beginning of the test, the stained pump oil was floating on water surface. Upon direct contact with the aerogel, the oil was quickly absorbed. After the completion of the absorption, the oil was completely removed and no stained oil was visibly detected on the water surface. The result suggests that the modified LC aerogel could effectively absorb oil from water surface, offering an environmentallyfriendly alternative option for oil spill cleaning.

$\mathrm{RH}$ residue after IL treatment was rinsed by DI water and dried in an oven. After that, the residue was calcined at $700{ }^{\circ} \mathrm{C}$ for $2 \mathrm{~h}$ to synthesize silica $[1,9,11,12]$. The XRD patterns shown in Fig. 5a indicate that the silica derived from the IL treated $\mathrm{RH}$ residue (RH-IL-silica) remained to be amorphous. It exhibits a similar XRD pattern as the silica derived from $\mathrm{HCl}$ treated $\mathrm{RHs}(\mathrm{RH}-$ $\mathrm{HCl}$-silica), a widely adopted process to synthesize silica from RHs [18]. Figure 5b shows the SEM image of RHIL-silica, which exhibits as nanoparticles with a diameter of ca. $40-80 \mathrm{~nm}$. Considering IL treated RH residue is a byproduct, using this residue to prepare silica contributes to the comprehensive application strategy of $\mathrm{RH}$ biomass, which improves the utilization efficiency of RHs and broadens the applications of RH biomass [18]. Due to the amorphous phase and relative high surface area, the silica derived from RHs can be used as a filler in plastics, rubbers, and personal care products, and as the starting material for various silicon-containing materials $[10]$.
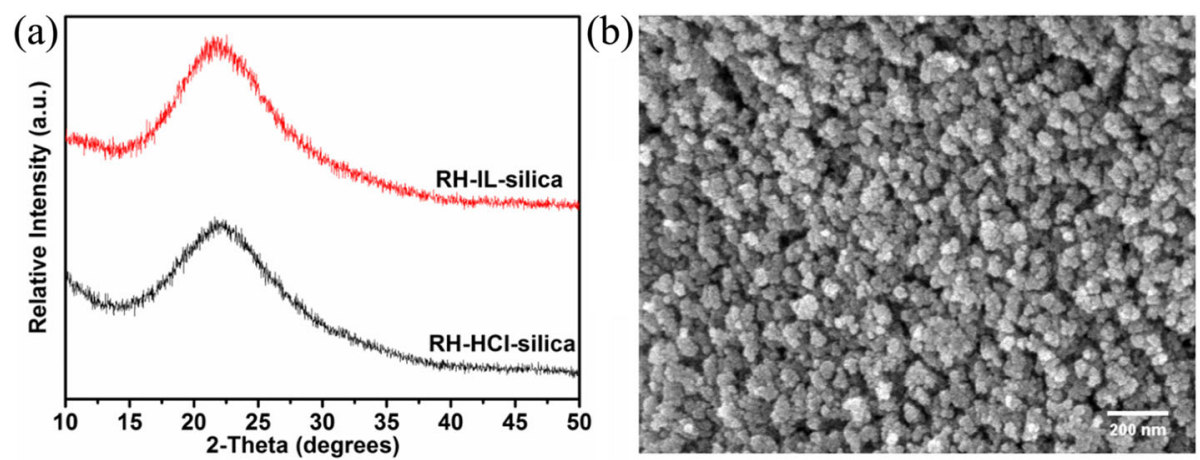

Fig. 5 a XRD patterns of the silica samples synthesized from IL treated RH residue and $\mathrm{HCl}$ treated raw RHs; $\mathbf{b}$ SEM image of the silica synthesized from IL treated $\mathrm{RH}$ residue 


\section{Conclusion}

In summary, the LC in RHs could be successfully dissolved into IL (BMIMCl) to form a homogenous solution. LC aerogel with a 3-D porous network structure was prepared by treating the LC IL solution by the NFT process followed by freeze drying. After the MTMS vapor treatment, the LC aerogel turned from hydrophilic to hydrophobic. Such a modified LC aerogel could potentially find uses in absorption applications, such as cleaning oil spills. The LC aerogel can be easily converted to carbon aerogel with a high surface area through a facile pyrolysis process. Meanwhile, amorphous silica nanoparticles could be prepared from the IL treated RH residue, leading to a comprehensive utilization of RH biomass.

\section{Supplementary information}

Supplementary information accompanies this paper at https://doi.org/10. 1186/s42825-020-00044-X.

Additional file 1. The experimental part is available in the supporting information.

\section{Abbreviations}

RHs: Rice Husks; BMIMCl: 1-butyl-3-methylimidazolium chloride; NFT: Nitrogen freeze-thaw; LC: Lignocellulose; ILs: Ionic liquids; CVD: Chemical vapor deposition; MTMS: Methyltrimethoxysilane; FESEM: Field emission scanning electron microscope; XRD: X-ray diffraction; BET: Brunauer-Emmett-Teller; SSA: Specific surface area; WCAs: Water contact angles; $\mathrm{RH}-\mathrm{HCl}$-silica: Silica derived from $\mathrm{HCl}$ treated $\mathrm{RH}$

\section{Acknowledgements}

Not applicable.

\section{Authors' contributions}

LS and ZW designed the project. ZW led the project, and ATS, WRTT, JL, and $\mathrm{DH}$ participated in the characterizations. HW and WW provided valuable suggestions to optimize the synthesis route. LS and HW co-supervised the project. ZW wrote the first draft. LS, ATS and HW revised the manuscript. All authors read and approved the final manuscript.

\section{Funding}

Not applicable.

\section{Availability of data and materials}

Not applicable.

\section{Competing interests}

There is no competing interest to declare.

\footnotetext{
Author details

${ }^{1}$ Polymer Program, Institute of Materials Science, University of Connecticut, Storrs, CT 06269, USA. ²Department of Chemical \& Biomolecular Engineering, University of Connecticut, Storrs, CT 06269, USA. ${ }^{3}$ College of Materials Science and Engineering, South China University of Technology, Guangzhou 510640, Guangdong, China. ${ }^{4}$ Key Laboratory of Enhanced Heat Transfer and Energy Conservation of the Ministry of Education, School of Chemistry and Chemical Engineering, South China University of Technology, Guangzhou 510640, Guangdong, China. ${ }^{5}$ Department of Biomedical Engineering, University of Connecticut, Storrs, CT 06269, USA.
}

Received: 3 May 2020 Accepted: 2 October 2020

Published online: 15 January 2021

\section{References}

1. Wang Z, Yu J, Zhang X, Li N, Liu B, Li Y, Wang Y, Wang W, Li Y, Zhang L, Dissanayake S, Suib SL, Sun L. Large-scale and controllable synthesis of graphene quantum dots from rice husk biomass: a comprehensive utilization strategy. ACS Appl Mater Interfaces. 2016;8:1434-9.

2. Wang Z, Smith AT, Wang W, Sun L. Versatile nanostructures from rice husk biomass for energy applications. Angew Chem Int Ed. 2018;57:13722-34.

3. Sun L, Xiao M, Xiao P, Song J, Wang W, Zhang Y, Gong K. A preliminary study on rice husk filled polypropylene composite. MRS Proc. 2000;661:KK5.14.

4. Wang W, Martin JC, Zhang N, Ma C, Han A, Sun L. Harvesting silica nanoparticles from rice husks. J Nanopart Res. 2011;13:6981-90.

5. Wang W, Martin JC, Fan X, Han A, Luo Z, Sun L. Silica nanoparticles and frameworks from rice husk biomass. ACS Appl Mater Interfaces. 2012;4:977-81

6. Krishnarao RV, Godkhindi MM. Distribution of silica in rice husks and its effect on the formation of silicon carbide. Ceram Int. 1992;18:243-9.

7. Rahman IA, Riley FL. The control of morphology in silicon nitride powder prepared from rice husk. J Eur Ceram. 1989:5:11-22.

8. Seo ESM, Andreoli M, Chiba R. Silicon tetrachloride production by chlorination method using rice husk as raw material. J Mater Process Technol. 2003;141:351-6.

9. Wang W, Martin JC, Huang R, Huang W, Liu A, Han A, Sun L. Synthesis of silicon complexes from rice husk derived silica nanoparticles. RSC Adv. 2012; 2:9036-41.

10. Sun L, Gong K. Silicon-based materials from rice husks and their applications. Ind Eng Chem Res. 2001;40:5861-77.

11. Wang Z, Chen H, Xu L, Xu SQ, Gao CF, Oliphant AJ, Liu J, Lu Y, Wang W, Sun L. Synthesis and colour prediction of stable pigments from rice husk biomass. Green Mater. 2015:3:10-4.

12. Li Y, Lan JY, Liu J, Yu J, Luo Z, Wang W, Sun L. Synthesis of gold nanoparticles on rice husk silica for catalysis applications. Ind Eng Chem Res. 2015;54:5656-63.

13. Wang Z, Zeng S, Joshi GN, Smith AT, Zeng H, Wei Z, Yu X, Pokhrel M, Mao $Y$, Wang W. Design and fabrication of highly photoluminescent carbonincorporated silica from rice husk biomass. Ind Eng Chem Res. 2019;58: 4688-94.

14. Wang Z, Zeng S, Li Y, Wang W, Zhang Z, Zeng H, Wang W, Sun L. Luminescence mechanism of carbon-incorporated silica nanoparticles derived from rice husk biomass. Ind Eng Chem Res. 2017;56:5906-12.

15. Liu Y, Wang Z, Zeng $H$, Chen C, Liu J, Sun L, Wang W. Photoluminescent mesoporous carbon-doped silica from rice husks. Mater Lett. 2015;142:280-2.

16. Wang Z, Liu J, Wang W, Wei Z, Wang F, Gong P, Wang J, Li N, Liu B, Zhang Z, Wang W, Sun L. Photoluminescent carbon quantum dot grafted silica nanoparticles directly synthesized from rice husk biomass. J Mater Chem B. 2017:5:4679-89.

17. Wei Z, Wang Z, Tait WR, Pokhrel M, Mao Y, Liu J, Zhang L, Wang W, Sun L. Synthesis of green phosphors from highly active amorphous silica derived from rice husks. J Mater Sci. 2018;53:1824-32.

18. Chen H, Wang W, Martin JC, Oliphant AJ, Doerr PA, Xu JF, DeBorn KM, Chen C, Sun L. Extraction of lignocellulose and synthesis of porous silica nanoparticles from rice husks: a comprehensive utilization of rice husk biomass. ACS Sustain Chem Eng. 2012;1:254-9.

19. Saha BC. Lignocellulose biodegradation and applications in biotechnology. Lignocellulose biodegradation. ACS Symposium Series. 889: American Chemical Society; 2004. p. 2-34.

20. Wang W, Wang Z, Liu J, Peng Y, Yu X, Wang W, Zhang Z, Sun L. One-pot facile synthesis of graphene quantum dots from rice husks for $\mathrm{Fe}^{3+}$ sensing. Ind Eng Chem Res. 2018:57:9144-50.

21. Tsuen-Hsuin T. Paper and printing. ChemChemTechnol.; 1985. p. 1-2.

22. Yang Q, Fukuzumi H, Saito T, Isogai A, Zhang L. Transparent cellulose films with high gas barrier properties fabricated from aqueous alkali/urea solutions. Biomacromolecules. 2011:12:2766-71.

23. Poplin JH, Swatloski RP, Holbrey JD, Spear SK, Metlen A, Grätzel M, Nazeeruddin MK, Rogers RD. Sensor technologies based on a cellulose supported platform. Chem Commun. f2007;20:2025-27. 
24. Cai J, Kimura S, Wada M, Kuga S, Zhang L. Cellulose aerogels from aqueous alkali hydroxide-urea solution. ChemSusChem. 2008;1:149-54.

25. Ludueña L, Fasce D, Alvarez VA, Stefani PM. Nanocellulose from rice husk following alkaline treatment to remove silica. BioResources. 2011;6:1440-53.

26. Lee SH, Doherty TV, Linhardt RJ, Dordick JS. Ionic liquid-mediated selective extraction of lignin from wood leading to enhanced enzymatic cellulose hydrolysis. Biotechnol Bioeng. 2009;102:1368-76.

27. Zhu S, Wu Y, Chen Q, Yu Z, Wang C, Jin S, Ding Y, Wu G. Dissolution of cellulose with ionic liquids and its application: a mini-review. Green Chem. 2006:8:325-7.

28. Swatloski RP, Spear SK, Holbrey JD, Rogers RD. Dissolution of cellose with ionic liquids. J Am Chem Soc. 2002;124:4974-5.

29. Li J, Lu Y, Yang D, Sun Q, Liu Y, Zhao H. Lignocellulose aerogel from woodionic liquid solution (1-Allyl-3-methylimidazolium chloride) under freezing and thawing conditions. Biomacromolecules. 2011;12:1860-7.

30. Wu Z-S, Yang S, Sun Y, Parvez K, Feng X, Müllen K. 3D nitrogen-doped graphene aerogel-supported Fe3O4 nanoparticles as efficient electrocatalysts for the oxygen reduction reaction. J Am Chem Soc. 2012; 134:9082-5.

31. Aliev AE, Oh J, Kozlov ME, Kuznetsov AA, Fang S, Fonseca AF, Ovalle R, Lima MD, Haque MH, Gartstein YN. Giant-stroke, superelastic carbon nanotube aerogel muscles. Science. 2009;323:1575-8.

32. Hu Y, Tong $X$, Zhuo H, Zhong L, Peng X, Wang S, Sun R. 3D hierarchical porous $\mathrm{N}$-doped carbon aerogel from renewable cellulose: an attractive carbon for high-performance supercapacitor electrodes and CO2 adsorption. RSC Adv. 2016;6:15788-95.

33. Nguyen ST, Feng J, Le NT, Le AT, Hoang N, Tan VB, Duong HM. Cellulose aerogel from paper waste for crude oil spill cleaning. Ind Eng Chem Res. 2013;52:18386-91.

34. Wan C, Li J. Facile synthesis of well-dispersed superparamagnetic $y$-Fe2O3 nanoparticles encapsulated in three-dimensional architectures of cellulose aerogels and their applications for $\mathrm{Cr}(\mathrm{VI})$ removal from contaminated water. ACS Sustain Chem Eng. 2015;3:2142-52.

35. Jiao $Y$, Wan C, Li J. Synthesis of carbon fiber aerogel from natural bamboo fiber and its application as a green high-efficiency and recyclable adsorbent. Mater Des. 2016;107:26-32

36. Zhai T, Zheng Q, Cai Z, Xia H, Gong S. Synthesis of polyvinyl alcohol/ cellulose nanofibril hybrid aerogel microspheres and their use as oil/solvent superabsorbents. Carbohydr Polym. 2016;148:300-8.

37. Wan C, Lu Y, Jiao Y, Jin C, Sun Q, Li J. Ultralight and hydrophobic nanofibrillated cellulose aerogels from coconut shell with ultrastrong adsorption properties. J Appl Polym Sci. 2015;132:42037.

38. Wang W-K, Tang B, Liu J, Shi H, Xu Q, Zhao G. Self-supported microbial carbon aerogel bioelectrocatalytic anode promoting extracellular electron transfer for efficient hydrogen evolution. Electrochim Acta. 2019;303:268-74.

39. Bi H, Yin Z, Cao X, Xie X, Tan C, Huang X, Chen B, Chen F, Yang Q, Bu X, Lu $X$, Sun $L$, Zhang $H$. Carbon fiber aerogel made from raw cotton: a novel, efficient and recyclable sorbent for oils and organic solvents. Adv Mater. 2013:25:5916-21.

40. Wu ZY, Li C, Liang HW, Chen JF, Yu SH. Ultralight, flexible, and fire-resistant carbon nanofiber aerogels from bacterial cellulose. Angew Chem. 2013;125: 2997-3001.

41. Ding B, Cai J, Huang J, Zhang L, Chen Y, Shi X, Du Y, Kuga S. Facile preparation of robust and biocompatible chitin aerogels. J Mater Chem. 2012;22:5801-9.

42. Lu Y, Sun Q, Yang D, She X, Yao X, Zhu G, Liu Y, Zhao H, Li J. Fabrication of mesoporous lignocellulose aerogels from wood via cyclic liquid nitrogen freezing-thawing in ionic liquid solution. J Mater Chem. 2012;22:13548-57.

43. Fratzl P. Cellulose and collagen: from fibres to tissues. Curr Opin Colloid Interface Sci. 2003;8:32-9.

44. Li J, Wang X, Huang Q, Gamboa S, Sebastian P. Studies on preparation and performances of carbon aerogel electrodes for the application of supercapacitor. J Power Sources. 2006;158:784-8.

45. Miller J, Dunn B, Tran T, Pekala R. Deposition of ruthenium nanoparticles on carbon aerogels for high energy density supercapacitor electrodes. J Electrochem Soc. 1997;144:L309-L11.

46. Chien HC, Cheng WY, Wang YH, Lu SY. Ultrahigh specific capacitances for supercapacitors achieved by nickel cobaltite/carbon aerogel composites. Adv Funct Mater. 2012;22:5038-43.

47. Moreno-Castilla C, Maldonado-Hódar F. Carbon aerogels for catalysis applications: an overview. Carbon. 2005;43:455-65.
48. Biener J, Stadermann M, Suss M, Worsley MA, Biener MM, Rose KA, Baumann TF. Advanced carbon aerogels for energy applications. Energy Environ Sci. 2011:4:656-67

49. Xu X, Zhou J, Nagaraju DH, Jiang L, Marinov VR, Lubineau G, Alshareef HN, Oh M. Flexible, highly graphitized carbon aerogels based on bacterial cellulose/lignin: catalyst-free synthesis and its application in energy storage devices. Adv Funct Mater. 2015;25:3193-202.

50. Kalita E, Nath B, Deb P, Agan F, Islam MR, Saikia K. High quality fluorescent cellulose nanofibers from endemic rice husk: isolation and characterization. Carbohydr Polym. 2015;122:308-13.

51. Sun $X$, Chi Y, Mu T. Studies on staged precipitation of cellulose from an ionic liquid by compressed carbon dioxide. Green Chem. 2014;16:2736-44.

52. Tang S, Baker GA, Ravula S, Jones JE, Zhao H. PEG-functionalized ionic liquids for cellulose dissolution and saccharification. Green Chem. 2012;14: 2922-32.

53. Cheng G, Varanasi P, Li C, Liu H, Melnichenko YB, Simmons BA, Kent MS, Singh S. Transition of cellulose crystalline structure and surface morphology of biomass as a function of ionic liquid pretreatment and its relation to enzymatic hydrolysis. Biomacromolecules. 2011;12:933-41.

54. Li W, Sun N, Stoner B, Jiang X, Lu X, Rogers RD. Rapid dissolution of lignocellulosic biomass in ionic liquids using temperatures above the glass transition of lignin. Green Chem. 2011:13:2038-47.

55. Gani A, Naruse I. Effect of cellulose and lignin content on pyrolysis and combustion characteristics for several types of biomass. Renew Energy. 2007;32:649-61.

56. Xiao S, Gao R, Lu Y, Li J, Sun Q. Fabrication and characterization of nanofibrillated cellulose and its aerogels from natural pine needles. Carbohydr Polym. 2015;119:202-9.

\section{Publisher's Note}

Springer Nature remains neutral with regard to jurisdictional claims in published maps and institutional affiliations.

\section{Submit your manuscript to a SpringerOpen ${ }^{\circ}$ journal and benefit from:}

- Convenient online submission

- Rigorous peer review

- Open access: articles freely available online

- High visibility within the field

Retaining the copyright to your article

Submit your next manuscript at $>$ springeropen.com 